\section{Clinical advances in degenerative dementias}

BRUCE L. MILLER

Until recently there was widespread nihilism in the clinical approach to the demented patient. Most physicians believed that there was little reason to spend money or effort diagnosing diseases with no known risk factors, preventive measures or treatments. However, a multi-disciplinary effort has transformed this field, leading to a better understanding of the clinical presentation, risk factors, pathogenesis and treatment of the three main degenerative dementias: Alzheimer's disease (AD), fronto-temporal dementia (FTD) and dementia with Lewy bodies (DLB). These advances have evolved so quickly that even the physician with a primary focus upon dementia may have difficulty keeping up with the changing terminology, new risk factors, better diagnostic strategies and emerging therapies.

Alongside the new discoveries related to the pathogenesis of various dementia syndromes, there have been steady advances in the clinical diagnosis of $A D$, FTD and DLB. The ability to diagnose and separate these different dementias accurately has advanced both basic and clinical research. Additionally, the diagnostic advances in dementia described below are beginning to transform the clinical approach to elderly patients with neurological or psychiatric impairment.

\section{ALZHEIMER'S DISEASE}

Almost 30 years ago, in this journal, Blessed et al (1968) provided the first definitive proof that amyloid plaques and neurofibrillary tangles correlated with dementia severity in the elderly. Their observations were based on the meticulous recording of mental status in elderly patients, upon whose death the brain concentration of amyloid plaques was determined. The authors found a strong correlation between dementia severity and brain amyloid. Six decades before, Alois Alzheimer had described similar pathological abnormalities in the brains of two pre-senile dementia patients. Suddenly, what had been called 'arteriosclerotic dementia' became Alzheimer's disease. Soon afterwards, a cholinergic deficit was discovered in association with AD (Bowen et al, 1976; Davies \& Malony, 1976; Perry et al, 1977).

These studies represented a paradigm shift by showing that elderly subjects with cognitive impairment were demented as a result of specific neurochemical and pathological changes in the brain. Subsequently, formal research criteria were established for the diagnosis of $\mathrm{AD}$, which required both the quantification of mental status (McKhann et $a l, 1984)$ and counting of amyloid plaques and neurofibrillary tangles. Emphasis upon quantifying mental status improved diagnostic accuracy for dementia. Simultaneously, $\mathrm{AD}$ research became directed toward determining the function, mechanisms of formation and neuronal toxicity of B-amyloid. The discovery of a cholinergic deficit eventually led to the development of cholinergic drugs with some efficacy against $A D$.

More recently, genetic research helped better to define the relationship between amyloid and AD. Chromosome 21 was found to be the site for the amyloid precursor protein gene (APP), and specific mutations adjacent to or involving $A P P$ were discovered in families with early-onset AD (Goate et al, 1991). Subsequently, apolipoprotein E4, which was encoded on chromosome 19, was found to be a strong risk factor for $\mathrm{AD}$ (Roses, 1994). Of the three haplotypes for the apolipoprotein $\mathrm{E}$ (E2, 3 and 4), E4 had the greatest avidity for amyloid, suggesting that abnormal processing of brain amyloid might explain the higher risk associated with E4. Although basic research into the relationship between apolipoprotein $\mathrm{E}$ and $\mathrm{AD}$ continues, apolipoprotein E4 lacks sensitivity and specificity as a screening tool for $\mathrm{AD}$.

Other genes were found. Many earlyonset $\mathrm{AD}$ cases were discovered to have an abnormality in the 'presenilin' protein encoded on chromosome 14 (Schellenberg, 1995). Also, chromosome 1 encodes a protein partially homologous with presenilin 1 (presenilin 2) linked to some familial cases of AD (Rogaev et al, 1995). The functions of these two proteins are under active study.

Unfortunately, many patients with non$A D$ degenerative dementias fulfil the current research criteria for AD. Not surprisingly, this has led to unacceptably high misdiagnosis rates for disorders such as FTD and Parkinsonian dementias. Mendez et al (1993) reported that 18 out of 21 patients with Pick's disease at autopsy were diagnosed as having AD during their life. Similarly, Parkinsonian dementia accounted for many of the patients misdiagnosed at $\mathrm{AD}$ diagnostic centres (Galasko et al, 1994). Recently, clinical pathological studies into FTD and Parkinson's disease led to the realisation that both disorders were clinically distinctive and could be separated from AD during life.

\section{FRONTO-TEMPORAL DEMENTIA}

Longitudinal studies from the research groups in Lund, Sweden and Manchester, England suggested that more than $15 \%$ of patients with degenerative dementia had a disorder which selectively attacked the anterior frontal and temporal cortex (Brun, 1987; Neary et al, 1988). The typical age of onset was the sixth decade and many FTD subjects had a history suggesting a late-life onset dominantly inherited disorder (Gustafson, 1993). Some patients developed motor neuron disease and others had relatives with a history of motor neuron disease (Mitsuyama, 1993). Accurate diagnosis of FTD required careful observation of behaviour because mental status testing alone was not sufficient (Gustafson, 1987; Neary et al, 1988). Functional imaging with single photon emission computed tomography (SPECT) showed selective fronto-temporal hypoperfusion and helped improve diagnostic accuracy for FTD (Read et al, 1995).

Pathologically distinct from $\mathrm{AD}$, frontal and temporal cortex demonstrated marked gliosis and neuronal loss, but only a minority had Pick bodies (Brun, 1987). Unlike AD, FTD was not associated with a pre-synaptic cholinergic deficit, although severe pre- and post-synaptic serotonin losses were found (Sparks \& Markesbery, 1991). 
The Lund and Manchester groups set research criteria for FTD, which consisted of 29 items divided into the catagories: behaviour, speech, affect, spatial orientation/ praxis, physical signs, investigations and supportive findings (Brun et al, 1994). Some of these items strongly distinguish FTD from $\mathrm{AD}$, including disinhibition, loss of personal awareness, hyper-orality, stereotyped and perseverative behaviour, progressive reduction of speech, and preserved spatial orientation (Miller et al, 1997). Neuropsychological tests distinguish FTD patients from healthy controls. However, they do not easily differentiate FTD patients from those with AD (Pahana et al, 1996).

FTD offers a fascinating model for understanding the clinical manifestations of selective anterior frontal or temporal dysfunction. Symptoms reflect the relative involvement of the left or right frontal or temporal lobes. Left-sided dysfunction causes loss of speech and language (Snowden et al, 1992), while selective right-sided degeneration commonly leads to behavioural disinhibition and altered expression of affect (Miller et al, 1993). Stereotyped compulsive behaviours and increased eating may be secondary to the serotonin deficit in FTD (Miller et al, 1995). Treatment with serotonin-boosting antidepressants has demonstrated promise of ameliorating some of the behaviours found in these patients, although they have shown no effect on the cognitive disturbance. Preventive measures seem unlikely until there is a better understanding of the molecular basis for this disorder. However, recent demonstration of linkage between familial cases of FTD and chromosome 17 suggests that the molecular mechanisms associated with FTD may soon be clarified (Wilhelmsen et al, 1994).

\section{DEMENTIA WITH LEWY BODIES}

This term was chosen at a recent international meeting which established research criteria for the group of patients who present with a mixture of dementia and Parkinsonism (McKeith et al, 1996). Whether these patients suffer from a variant of $\mathrm{AD}$ (Hansen et al, 1990) or have a distinct disorder (Perry et al, 1990a,b; Dickson et al, 1991) is still debated, but it is accepted that clinical and pathological findings are distinctive in DLB.

Studies from England (Byrne et al, 1989; Perry et al, 1990b) and Japan (Kosaka,
1990) were the first to emphasise the distinctive clinical features of DLB patients. In many, visual hallucinations occur (McKeith et al, 1996), often featuring complex scenes with animals, dwarves, children or supernatural creatures. Neurochemical studies documented a profound cholinergic deficit in DLB and suggested that the visual hallucinations and fluctuating mental status characteristic of this disorder might have a chemical basis (Perry et al, $1990 a$ ). Parkinsonian features, often different from classical Parkinson's disease, have been reported in most DLB patients and can precede or follow the onset of dementia (McKeith et al, 1996). Postural instability and bradykinesia are common, while tremor is rare. Neuropsychological and neuroimaging studies have not shown robust differences between DLB and AD subjects (Burns, 1996). DLB patients have Lewy bodies in the substantia nigra and cortex (Perry et al, $1990 b$ ) and show depletion of dopamine in the substantia nigra. Amyloid plaques are present in some but not all subjects.

Diagnosis of DLB has therapeutic implications; because of the dopaminergic deficiency, treatment with neuroleptics can be fatal, while anticholinergic medications often precipitate life-threatening delirium (McKeith et al, 1996). Also, DLB patients may be more likely to respond to anticholinesterases than those with classical AD (Kaufer \& Cummings, 1996).

\section{CONCLUSION}

With the steady march of important discoveries in dementia, including the emergence of treatment options, recognition of the clinical features of the different degenerative dementias has become particularly important. Despite the many basic scientific advances related to $\mathrm{AD}$, FTD and DLB, diagnosis still requires hands-on evaluation of the patient, which must include meticulous recording of the history, testing of mental status and observation and quantifi= cation of psychiatric behaviours. Rather than being pushed aside by molecular biology, clinical neuropsychiatry has become central to the diagnosis and treatment of the major degenerative disorders.

\section{REFERENCES}

Blessed, G., Tomlinson, B. E. \& Roth, M. (1968) The association between quantitative measures of dementia and of senile change in the cerebral grey matter of elderly subjects. British Journal of Psychiatry. 114. 797-811.

Bowen, D. M., Smith, C. B., White, P., ef al (1976) Neurotransmitter-related enzymes and indices of hypoxia in senile dementia and other abiotrophies. Brain. 99, 459-496.

Brun, A. (1987) Frontal lobe degeneration of non-Alzheimer type. I: Neuropathology. Archives of Gerontology and Geriatrics. 6. 193-208.

—. Englund, B., Gustafson, L., et al (1994)

Clinical and neuropathological criteria for frontotemporal dementia. Journal of Neurology. Neurosurgery and Psychiatry. 57. 416-418.

Burns, A. (1996) Clinical features of patients with Alzheimer's disease and Lewy bodies. In Dementia with Lewy Bodies (eds R. Perry. I. Mc Keith \& E. Perry). pp. 46-56. Cambridge: Cambridge University Press.

Byrne, E. J., Lennox, G., Lowe, J., et al (1989) Lewy Body disease. clinical features in 15 cases. Journal of Neurology. Neurosurgery and Psychiotry, 52. 709-717.

Davies, P. \& Malory, A. J. F. (1976) Selective loss of central cholinergic neurons in Alzheimer's disease. Lancet, II, 1403.

Dickson, D. W., Ruan, D., Crystal, H., et al (1991) Hippocampal degeneration differentiates diffuse Lewy body disease (DLBD) from Alzheimer's disease: light and electron microscopic immunohistochemistry of CA 2-3 neurites specific to DLBD. Neurology. 41, 1402-1409.

Galasko, D., Hansen, L. A., Katzman, R., et ol (1994) Clinical - neuropathological correlations in Alzheimer's disease and related dementias. Archives of Neurology. 51. 888-895

Goate, A., Chartier-Harlin, M. C., Mullan, M., et ol (1991) Segregation of a missense mutation in the amyloid precursor protein gene with familial Alzheimer's disease. Nature, 349. 704-706.

Gustafson, L. (1987) Frontal lobe degeneration of nonAlzheimer type. II: Clinical picture and differential diagnosis. Archives of Gerontology and Geriotrics, 6. 209-223.

- (1993) Clinical picture of frontal lobe degeneration of non-Alzheimer type. Dementıo, 4, 143-148.

Hansen, L., Salmon, D., Galasko, D., ef of (1990) The Lewy body variant of Alzheimer's disease: A clinical and pathological entity. Neurology, 40, 1-8.

Kaufer, D. \& Cummings, J. L. (1996) Neuropsychiatric aspects of Alzheimer's disease: The cholinergic hypothesis revisited. Neurology, 47, 87I-875.

Kosaka, K. (1990) Diffuse Lewy body disease in Japan. Journo of Neurology. 237. 197-204.

McKeith, I. G., Galasko, D., Kosaka, K., et ol (1996) Clinica and pathological diagnosis of dementia with Lewy bodies (DLB): Report of the CDLB international workshop. Neurology. 47. $1113-1125$

MeKhann, G., Drachman, D., Folstein, M. F., et of (1984) Clinical diagnosis of Alzheimer's disease: report of the NINCDS-ADRDA Work Group under the auspices of the Department of Health and Human Services Task Force on Alzheimer's disease. Neurology, 34, 939-944.

BRUCE L. MILLER, MD. Department of Neurology F-9. Harbor-UCLA Medical Center, 1000 West Carson Street. Torrance. California 90509. USA

(First received 31 January 1997, accepted 25 March 1997) 
Mondez, M. F., Selwood, A., Mastri, A. F., et ol (1993) Pick's disease versus Alzheimer's disease: a comparison of clinical characteristics. Neurology, 43, 289--292.

Miller, B. L., Chang, L., Mena, I., et al (1993) Clinical and imaging features of right focal frontal lobe degenerations. Dementia. 6. 195-199.

_, Darby, A. L., Swartz, J. R., et ol (1995) Dietary changes, compulsions and sexual behavior in fronto-tempora degeneration. Dementia, 6. 195-199.

_. Ikonte, C., Ponton, M. P., et al (1997) The usefulness of the Lund-Manchester research criteria for frontotemporal dementia. Journal of the International Neuropsychological Society 2. 505-510.

Mitsuyama, Y. (1993) Presenile dementia with motor neuron disease. Dementio, 4, 137-142.

Neary, D., Snowden, J. S., Northen, B., et ol (1988)

Dementia of frontal lobe type. Journal of Neurology.

Neurosurgery and Psychiatry, 51. 353-361.

Pahana, N. A., Boone, K. B., Miller, B. L., et al (1996)

Comparison of neuropsychological functioning in Alzheimer's disease and frontotemporal dementia. Journol of the International Neuropsychological Society. 2. 505-510.

Perry, E. K., Perry, R. H., Blessed, G., et al (1977) Necropsy evidence of central cholinergic deficits in senile dementia. Lancet, 1, 189

_. Marshall, E., Kerwin, J., et ol (19900) Evidence of monoaminergic - cholinergic imbalance related to visual hallucinations in Lewy body dementia. Journal of Neurochemistry, 55, 1454-1456.

Perry, R. H., Irving, D., Blessed, G., et al (1990b) Senile dementia of Lewy body type. Journal of Neurological Sciences. 95. 119-139.

Read, S., Miller, B. L., Mena, l., et al (1995) SPECT in dementia: Clinical and pathological correlation. Journa of the American Geriatric Society, 43. 1243-1247.

Rogaev, E., Sherrington, R., Liang, Y., et ol (1995) Familia Alzheimer's disease in kindreds with missense mutations in a gene on chromosome I related to the Alzheimer's disease type 3 gene. Nature, 376, 600-602.
Roses, A. D. (1994) Apolipoprotein $E$ affects the rate of Alzheimer disease expression: beta-amyloid burden is a secondary consequence dependent on APOE genotype and duration of disease. Journal of Neuropathology and Experimental Neurology, 53, 429-437.

Schellenberz, G. D. (1995) Genetic dissection of Alzheimer disease: a heterogeneous disorder. Proceedings of the National Academy of Sciences of the United States of America. 92. 8552-8559.

Snowden, J. S., Neary, D., Mann, D. M. A., et ol (1992) Progressive language disorder due to lobar atrophy. Annals of Neurology, 31, 174-183.

Sparks, D. L. \& Markesbery, W. R. (1991) Altere serotonergic and cholinergic synaptic markers in Pick's disease. Archives of Neurology, 48. 796-799.

Wilhelmsen, K., Lynch, T., Paviou, E., ex al (1994) Localization of disinhibition-dementia - parkinsonism amyotrophy complex to 17q21-22. Americon journal of Human Genetics, 55. $1150-1165$ 